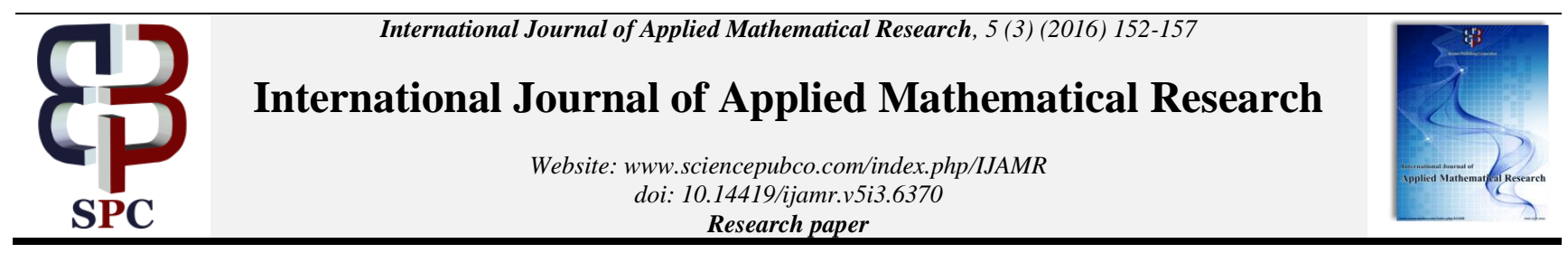

\title{
New efficiency algorithm for solving fractional order differential-algebraic system
}

\author{
Sameer Qasim Hasan ${ }^{1 *}$, Eman Mohammed Nemah ${ }^{2}$ \\ ${ }^{1}$ Department of Mathematics College of education University of Almustansryah, Iraq \\ ${ }^{2}$ Department of Mathematics College of education for Pure Sciences Ibn Al-Haitham University of Baghdad, Iraq \\ *Corresponding author E-mail: dr.Sameer_Kasim@yahoo.com
}

\begin{abstract}
This work provided the evolution of the algorithm for analytic solution of system of fractional differential-algebraic equations (FDAEs). The algorithm referred to good effective method for combination the Laplace Iteration method with general Lagrange multiplier (LLIM). Through this method we have reached excellent results in comparison with exact solution as we illustrated in our examples.
\end{abstract}

Keywords: Analytic Solution; Laplace Iteration Method; Laplace Transform; Invers Laplace Transform; System of Fractional Order DifferentialAlgebraic Equations.

\section{Introduction}

The fractional calculus has a long history from 30 September 1695, when the derivative of $\alpha=\frac{1}{2}$ has been described by Leibniz [1], [2]. The theory of derivatives and integrals of non-integer order goes back to Leibniz, Liouville, Grunwald, Letnikov and Riemann. There are many interesting books about fractional calculus and fractional differential equations [1], [2], [3], [4].

Differential equations of fractional order have been found to be effective to describe some physical phenomena such as rheology, damping laws, fluid flow and so on [5], [6].

Recently, many important mathematical models can be expressed in terms of systems of fractional order differential-algebraic equations.

In general, most fractional order differential-algebraic equations do not have exact solution. Therefore, development of effective numerical techniques, which offer precise approximate solution, has become an active research area. In this regard, Ibis and Bayram [7], Ibis et al. [8], Zurigat et al. [9], and Ding and Jiang [10], Damarla S.K., and M. Kundu [11], Barani B., et al.[12], extended the application of Adomian Decomposition Method (ADM), Variational Iteration Method (VIM), Fractional Differential Transform Method (FDTM), Homotopy Analysis Method (HAM), Homotopy Perturbation Method (HPM), Generalized Triangular Function Operational Matrices (TF), and Waveform relaxation Method to solve fractional order differential -algebraic equations.

In 2013, Habibolla et al. [13], were used the Laplace Iteration method (LIM) techniques to find approximation solution to the system of linear and nonlinear equations it did not require any small parameter in an equation as perturbation techniques do and use a Lagrange multiplier, and which called Reconstruction Variational Iteration method.

This paper is concerned with the development of an efficient algorithm for the analytic solutions of systems of fractional differential-algebraic equations (FDAEs). The proposed algorithm is an elegant combination of the Laplace Iteration method with the La- grange multiplier (LLIM). Moreover, it was demonstrated to solve a large class of linear and nonlinear system problems effectively, easily and accurately. Using approximation which rapidly, converge to accurate solutions.

The paper is organized as follows: In section 2, we give the definitions of fractional calculus. In section 3, we now identification of the Lagrange multipliers. We give a brief description of how the method works and propose an algorithm with Lagrange multiplier. LIM for system of fractional order differential-algebraic equations, in section 4 . We applied the algorithm on some linear and nonlinear FDAEs in section 5. Finally, we give some concluding remarks in section 6 .

\section{Basic definitions of fractional calculus}

In this section, we provide used definitions of fractional calculus [14].

Definition 2.1: A real function $f(t), t>0$, is said to be in the space $C_{\mu}, \mu \in R$ if there exists a real number $p>\mu$, such that $f(t)=$ $t^{p} f_{1}(t)$, where $f_{1}(t) \in C[0, \infty]$ and it is said to be in the space $C_{\mu}^{m}$ if and only if $f^{(m)} \in C_{\mu}, m \in N$.

Definition 2.2: The Riemann-Liouville fractional order integral of $\alpha>0$ of function $f(t) \in C_{\mu}, \mu>-1$ is defined as

$J^{\alpha} f(t)=\frac{1}{\Gamma(\alpha)} \int_{0}^{t}(t-\tau)^{\alpha-1} f(\tau) d \tau$

Definition 2.3: The Riemann-Liouville fractional order derivative (RL) of function $\mathrm{f}(\mathrm{t})$ is defined as

$$
\begin{aligned}
& D_{t}^{\alpha} f(t)=D^{m} J^{m-\alpha} f(t) \\
& =\frac{1}{\Gamma(m-\alpha)} \frac{d^{m}}{d t^{m}} \int_{0}^{t}(t-\tau)^{m-\alpha-1} f(t) d \tau, t>0
\end{aligned}
$$


Where $\alpha$ is a non-integer satisfying the relation $m-1<\alpha \leq$ $\mathrm{m}, \mathrm{m} \in \mathrm{N}$.

Definition 2.4: The fractional order derivative $(R L)$ of function $f(t)$ in Caputo sense is defined as

$\mathrm{D}_{*}^{\alpha} \mathrm{f}(\mathrm{t})=\mathrm{J}^{\mathrm{m}-\alpha} \mathrm{D}^{\mathrm{m}} \mathrm{f}(\mathrm{t})$

$=\frac{1}{\Gamma(m-\alpha)} \int_{0}^{t}(t-\tau)^{m-\alpha-1} f^{(m)}(\tau) d \tau, t>0$

For the Caputo's derivative we have,

$\mathrm{D}^{\alpha} \mathrm{c}=0, \mathrm{c}$ constant

$D^{\alpha} t^{\beta}=\left\{\begin{array}{c}0, \beta \leq \alpha-1 \\ \frac{\Gamma(\beta+1)}{\Gamma(\beta-\alpha+1)} t^{\beta-\alpha}, \beta>\alpha-1\end{array}\right.$

Caputos fractional differentiation is a linear operation and if $\mathrm{f}(\tau)$ is continuous in $[\mathrm{a}, \mathrm{t}]$ and $\mathrm{g}(\tau)$ has $\mathrm{m}+1$ continuous derivatives in $[a, t]$, it satisfies the so called Leibnitz rule

$$
D^{\propto}(f(t) g(t))=\sum_{k=0}^{\infty}\left(\begin{array}{l}
\propto \\
k
\end{array}\right) g^{(k)}(t) D^{\alpha-k} f(t)
$$

For establishing our results.

Definition 2.5: The single parameter and the two parameters variants of the Mittag- Leffler function are denoted by $E_{\alpha}(t)$ and $E_{\propto, \beta}(t)$, respectively, which are relevant for their connection with fractional calculus, and are defined as:

$$
\begin{aligned}
& E_{\propto}(t)=\sum_{k=0}^{\infty} \frac{t^{k}}{\Gamma(\alpha k+1)}, \propto>0, t \in C \\
& E_{\propto, \beta}(t)=\sum_{k=0}^{\infty} \frac{t^{k}}{\Gamma(\alpha k+\beta)}, \propto, \beta>0, t \in C
\end{aligned}
$$

Some special cases of the Mittag-Leffler function are as follows:

$E_{1}(t)=e^{t}, E_{\propto, 1}(t)=E_{\alpha}(t)$.

Other properties of the Mittag-Leffler functions can be found in [15]. These functions are generalizations of the exponential function because; most linear differential equations of fractional order have solution that are expressed in terms of these functions.

\section{Variational iteration method}

To illustrate the basic concept of the technique, we consider the following general differential equation:

$\mathrm{L}(\mathrm{x})+\mathrm{N}(\mathrm{x})-\mathrm{f}(\mathrm{t})=0$,

Where $\mathrm{L}$ is a linear operator, $\mathrm{N}$ is a nonlinear operator, and $\mathrm{f}(\mathrm{t})$ the function term. In the variational iteration method [16], [17] [18], [19], a correction functional can be constructed as follows:

$$
x_{n+1}(t)=x_{n}(t)+\int_{0}^{t} \lambda(s)\left(L x_{n}(s)+N \widetilde{x_{n}}(s)-f(s)\right) d s
$$

Where $\lambda(t)$ is a general Lagrange multiplier [16], [17],[18], [19], which can be identified optimally via a variational iteration method. The subscripts $\mathrm{n}$ denoted the $\mathrm{nth}$ approximation, $\widetilde{\mathrm{x}_{\mathrm{n}}}$ is considered as a restricted variation. That is $\overline{\delta x_{n}}=0$ : equation (3.2), is called a correct functional.

\section{Dsecription of the new method (LLIM)}

In this article, we consider the following non-homogenous, nonlinear system of fractional order differential-algebraic equations
$\left.\begin{array}{c}D_{*}^{\alpha_{i}} x_{i}(t)=h_{i}\left(t, x_{1}, x_{2}, \ldots, x_{n}\right) \\ 0=g\left(t, x_{1}, x_{2}, \ldots, x_{n}\right)\end{array}\right\}$

With initial conditions $x_{i}(0)=a_{i}, i=1,2, \ldots, n$. Here $D_{*}^{\alpha_{i}}$ is Caputo fractional derivative of order $\propto_{i}$, satisfying the relation

$m-1<\propto_{i} \leq m, m \in N$.

Eq. (4.1) can be rewritten as:

$$
\left.\begin{array}{c}
\mathrm{L}_{1} \mathrm{x}_{1}(\mathrm{t})+\mathrm{N}_{1}\left(\mathrm{t}, \mathrm{x}_{1}, \mathrm{x}_{2}, \ldots, \mathrm{x}_{\mathrm{n}}\right)=\mathrm{f}_{1}(\mathrm{t}) \\
\mathrm{L}_{2} \mathrm{x}_{2}(\mathrm{t})+\mathrm{N}_{2}\left(\mathrm{t}, \mathrm{x}_{1}, \mathrm{x}_{2}, \ldots, \mathrm{x}_{\mathrm{n}}\right)=\mathrm{f}_{2}(\mathrm{t}) \\
\cdot \\
\cdot \\
\mathrm{L}_{\mathrm{n}} \mathrm{x}_{\mathrm{n}}(\mathrm{t})+\mathrm{N}_{\mathrm{n}}\left(\mathrm{t}, \mathrm{x}_{1}, \mathrm{x}_{2}, \ldots, \mathrm{x}_{\mathrm{n}}\right)=\mathrm{f}_{\mathrm{n}}(\mathrm{t}) \\
0=\mathrm{g}\left(\mathrm{t}, \mathrm{x}_{1}, \mathrm{x}_{2}, \ldots, \mathrm{x}_{\mathrm{n}}\right)
\end{array}\right\}
$$

Where $L_{i}$ is a linear operator, $N_{i}$ a nonlinear operator and $f_{i}(t)$ is an inhomogenous item form $i=1,2 \ldots n$. Eq. (4.2) can be rewritten down as a correction function in the following way:

$L_{i} x_{i}(t)=f_{i}(t)-N_{i}\left(t, x_{1}, x_{2}, \ldots, x_{n}\right)$

$=\mathrm{R}_{\mathrm{i}}\left(\mathrm{t}, \mathrm{x}_{1}, \mathrm{x}_{2}, \ldots, \mathrm{x}_{\mathrm{n}}\right), \mathrm{i}=1,2 \ldots \mathrm{n}$

Therefore:

$L_{i} x_{i}(\mathrm{t})=R_{i}\left(t, x_{1}, x_{2}, \ldots, x_{n}\right), \mathrm{i}=1,2 \ldots \mathrm{n}$.

The Laplace Iteration Method assumed a series solution for $x_{i}$ given by an infinite sum of components:

$x_{i}(t)=\lim _{n \rightarrow \infty} x_{i}^{p}(t)=\lim _{n \rightarrow \infty} \sum_{j=0}^{p} v_{i}^{j}(t), i=1,2, \ldots, n$

In which $x_{i}^{n}$ indicates the n-th approximation of $x_{i}$, where $v_{i}^{j}$ is the $j^{\text {th }}$ component of the solution of $x_{i}$ and $v_{i}^{0}$ is the solution of $L_{i} x_{i}=0$ along with the following initial conditions of the main problem:

$v_{i}^{1}(t)=\varphi_{i}\left(v_{i}^{0}\right)$

$v_{i}^{k+1}(t)=\varphi_{i}\left(\sum_{j=0}^{k} v_{i}^{j}(t)\right)-\sum_{j=0}^{k} v_{i}^{j}(t), k \geq 1$

In which $\varphi_{i}\left(v_{i}^{k}\right)$ is obtained as follows:

$L_{i} \varphi_{i}\left(v_{1}^{k}, v_{2}^{k}, \ldots, v_{n}^{k}\right)=R_{i}\left(t, x_{1}, x_{2}, \ldots, x_{n}\right)$,

Using the homogenous initial conditions, supposing that $L_{i}=D^{\alpha_{i}}$, therefore, taking Laplace transform to both sides of Eq. (4.5) in the usual way and using the homogenous initial conditions, the result can be obtained as following:

$p_{i}(s) . \Phi_{i}^{k}(s)=\mathcal{R}_{i}\left(v_{i}^{k}(s)\right)$,

Where $\mathcal{L}\left[\varphi_{i}\left(v_{1}^{k}, v_{2}^{k}, \ldots, v_{n}^{k}\right)\right]=\Phi_{i}^{k}, p_{i}(s)$ is a fractional polynomial with the fractional degree of the highest derivative in Eq. (4.6) (The same as the highest order of the linear operator $L_{i}$ ). Thus,

$\mathcal{L}[w]=\varpi, \psi_{i}(s)=\frac{1}{p_{i}(s)}, \mathcal{L}\left[u_{i}(t)\right]=\psi_{i}(s)$

In Equations (4.5) and (4.6), the function $\mathcal{R}_{i}\left(v_{i}^{k}(s)\right)$ and $R_{i}\left(t, x_{1}, x_{2}, \ldots, x_{n}\right)$ are abbreviated as $\mathcal{R}_{i}$ and $R_{i}$ respectively. Hence, Eq. (4.6) is rewritten as:

$\Phi_{i}^{k}(s)=\mathcal{R}_{i}\left(\left(v_{1}^{k}, \mathrm{v}_{2}^{k}, \ldots, v_{n}^{k}\right)(s)\right) \cdot \psi_{i}(s)$ 
Now, by applying the inverse Laplace Transform to both side of Eq. (4.8) and using the convolution Theorem, the following relation can be presented:

$$
\begin{aligned}
& \emptyset_{i}\left(v_{1}^{k}, v_{2}^{k}, \ldots, v_{n}^{k}\right) \\
& =\int_{0}^{t} R_{i}\left(\left(v_{1}^{k}, v_{2}^{k}, \ldots, v_{n}^{k}\right)(\tau)\right) \cdot u_{i}(t-\tau) d \tau
\end{aligned}
$$

Therefore

$$
x_{i}^{p+1}(t)=\sum_{j=0}^{p+1} v_{i}^{j}(t)=x_{i}^{0}(t)+\int_{0}^{t} R_{i}\left(x_{i}^{p}(\tau)\right) \cdot u_{i}(t-\tau) d \tau
$$

After identifying the initial approximation of $x_{i}^{0}$, the remaining approximations $x_{i}^{p}, p>0$ can be determined so that each term can be determined by previous terms and the approximation of iteration formula can be entirely evaluated.

Consequently, the exact solution may be obtained by:

$$
\begin{aligned}
& x_{i}=\lim _{p \rightarrow \infty} x_{i}^{p}(t) \\
& =\lim _{p \rightarrow \infty} \sum_{j=0}^{p} v_{i}^{j}(t), i=1,2, \ldots, n
\end{aligned}
$$

Which is the Laplace Iteration method.

Now, we can construct a correct function as follows:

$$
\begin{aligned}
& x_{n+1}(t)=x_{0}(t)+ \\
& \int_{0}^{t} \lambda(\tau) R_{i}\left(\left(v_{1}^{k}, v_{2}^{k}, \ldots, v_{n}^{k}\right)(\tau)\right) \cdot u_{i}(t-\tau) d \tau
\end{aligned}
$$

The optimal value of the general Lagrange multipliers $\lambda$ can be identified by using the stationary conditions of the variational theory.

And now, consider nonlinear fractional order differential equation:

$$
D_{*}^{\alpha} x(t)+a x(t)+N x(t)-f(t)=0
$$

The correction function (4.13) can be approximately expressed a follows:

$$
x_{n+1}(t)=x_{n}(t)+{ }_{0} I_{t}^{\alpha}\left[\lambda(\tau)\left(D_{*}^{\alpha} x(t)+a x(t)+N x(t)-f(t)\right)\right]
$$

This yields the stationary conditions

$$
\begin{aligned}
\lambda(t)=-1 \text { and } \lambda^{(\alpha)}(t)-a \lambda(t) & =0 . \\
\lambda^{(\alpha)}(t)-a \lambda(t)=0 \Rightarrow \lambda(t) & =E_{\alpha, 1}\left(a t^{\alpha}\right) \lambda(0) \\
& =-\sum_{k=0}^{\infty} \frac{\left(a t^{\alpha}\right)^{k}}{\Gamma(\alpha k+1)}
\end{aligned}
$$

\section{Consider two cases:}

If $\mathrm{a}=1$ then $\lambda(t)=-\sum_{k=0}^{\infty} \frac{t^{\alpha k}}{\Gamma(\propto k+1)}$

If $\mathrm{a}=-1$ then $\lambda(t)=\sum_{k=0}^{\infty} \frac{(-1)^{k+1} t^{\alpha k}}{\Gamma(\alpha k+1)}$

\section{Applications and results}

In this section, we solve linear and nonlinear fractional order differential -algebraic equations by Laplace Iteration Method with Lagrange Multiplier.

Example 1: consider the following system of linear fractional order differential -algebraic equations [11]

$$
\left.\begin{array}{c}
D_{*}^{\alpha} x(t)+x(t)-y(t)+\sin t=0 \\
x(t)+y(t)=e^{-t}+\sin t, t \in[o, 1], 0<\alpha \leq 1
\end{array}\right\}
$$

Subject to initial conditions $\mathrm{x}(0)=1$, y $(0)=0$. For the special case of $\alpha=1$, we have analytical solution $x(t)=e^{-t}$ and $y(t)=$ $\sin t$.

Solution:

From the Eq. (14), optimal selection auxiliary linear operator the equation is represented as follows:

$L_{1} x(t): D_{*}^{\alpha} x(t)+x(t)=y(t)-\sin t$.

Therefore $\emptyset\left(v_{i}^{k}\right)$ is defined as:

$\varnothing\left(v_{1}^{k}, v_{2}^{k}\right)=\int_{0}^{t} u(t-\tau)\left[v_{2}^{k}-\sin t\right] d \tau$

Then, using Eq. (14), the Laplace Iteration Method with Lagrange Multiplier formulae in t-direction for the calculation of the approximate solution of Eq. (15) can be readily obtained as:

$$
\left.\begin{array}{c}
x_{n+1}(t)=x_{0}(t)+\int_{0}^{t}\left[\lambda(\tau) u(t-\tau)\left(y_{n}(\tau)-\sin t\right)\right] d \tau \\
y_{n+1}(t)=e^{-t}-x_{n+1}(t)+\sin t .
\end{array}\right\}
$$

\section{Case 1: $\alpha=1$}

$L_{1} x(t): D_{*}^{1} x(t)+x(t)=y(t)-\sin t$.

$\Rightarrow p(s)=\mathcal{L}\left[D_{*}^{\alpha} x(t)+x(t)\right]=s+1$

$\Rightarrow \psi(s)=\frac{1}{p(s)}=\frac{1}{s+1}$

$\Rightarrow u(t)=\mathcal{L}^{-1}[\psi(s)]=e^{-t}$

$u(t-\tau)=e^{(\tau-t)}$

Where the initial approximation must be satisfied by the following equations:

$L_{1} x(t)=0, x(0)=1$.

Therefore, it can be started by:

$x_{0}(t)=v_{1}^{0}=e^{-t}$

$y_{0}(t)=v_{2}^{0}=\sin t$

Accordingly, by Eq. (5.2) the higher order approximation of the exact solution can be obtained as follows:

$x_{1}(t)=\sum_{i=0}^{1} v_{1}^{k}(t)=e^{-t}$

$y_{1}(t)=\sum_{i=0}^{1} v_{2}^{k}(t)=\sin t$

The remaining approximations $x_{n}=0, y_{n}=0, n>1$ can be completely determined such that each term will be determined using the prevous term: thus, the exact solution is as follows:

$x(t)=\lim _{p \rightarrow \infty} \sum_{i=0}^{p} v_{1}^{k}(t)=e^{-t}$

$y(t)=\lim _{p \rightarrow \infty} \sum_{i=0}^{p} v_{2}^{k}(t)=\sin t$

Case 2: $\alpha=0.5$

$L_{1} x(t): D_{*}^{0.5} x(t)+x(t)=y(t)-\sin t$.

$\Longrightarrow p(s)=\mathcal{L}\left[D_{*}^{o .5} x(t)+x(t)\right]=s^{0.5}+1$

$\Rightarrow \psi(s)=\frac{1}{p(s)}=\frac{1}{s^{0.5}+1}$ 
$\Rightarrow u(t)=\mathcal{L}^{-1}[\psi(s)]=t^{-0.5} \sum_{k=0}^{\infty} \frac{(-\sqrt{t})^{k}}{\Gamma\left(\frac{k}{2}+\frac{1}{2}\right)}$

Where the initial approximation must be satisfied by the following equations:

$L_{1} x(t)=0, x(0)=1$.

Therefore, it can be started by:

$x_{0}(t)=v_{1}^{0}=E_{\frac{1}{2}, 1}(-\sqrt{t})=\sum_{k=0}^{\infty} \frac{(-\sqrt{t})^{k}}{\Gamma\left(\frac{k}{2}+1\right)}$

$y_{0}(t)=v_{2}^{0}=e^{-t}-\sum_{k=0}^{\infty} \frac{(-\sqrt{t})^{k}}{\Gamma\left(\frac{k}{2}+1\right)}+\sin t$

Accordingly, by Eq. (5.2) the higher order approximation of the exact solution can be obtained as follows:

$x_{1}(t)=\sum_{i=0}^{1} v_{1}^{k}(t)=\sum_{k=0}^{\infty} \frac{(-\sqrt{t})^{k}}{\Gamma\left(\frac{k}{2}+1\right)}+\int_{0}^{t}\left(\sum_{k=0}^{\infty} \frac{(-1) \tau^{\frac{k}{2}}}{\Gamma\left(\frac{k}{2}+1\right)}\right)((t-$

$\left.\tau)^{-0.5} \sum_{k=0}^{\infty} \frac{(-1)^{k}(t-\tau)^{\frac{k}{2}}}{\Gamma\left(\frac{k}{2}+\frac{1}{2}\right)}\right)\left(e^{-\tau}-\sum_{k=0}^{\infty} \frac{(-\sqrt{t})^{k}}{\Gamma\left(\frac{k}{2}+1\right)}\right) d \tau$

$y_{1}(t)=$

$\sum_{i=0}^{1} v_{2}^{k}(t)=e^{-t}-\sum_{k=0}^{\infty} \frac{(-\sqrt{t})^{k}}{\Gamma\left(\frac{k}{2}+1\right)}+\int_{0}^{t}\left(\sum_{k=0}^{\infty} \frac{(-1) \tau^{\frac{k}{2}}}{\Gamma\left(\frac{k}{2}+1\right)}\right)((t-$

$\left.\tau)^{-0.5} \sum_{k=0}^{\infty} \frac{(-1)^{k}(t-\tau)^{\frac{k}{2}}}{\Gamma\left(\frac{k}{2}+\frac{1}{2}\right)}\right)\left(e^{-\tau}-\sum_{k=0}^{\infty} \frac{(-\sqrt{t})^{k}}{\Gamma\left(\frac{k}{2}+1\right)}\right) d \tau+\sin t$

\section{Case 3: $\alpha=0.75$}

$L_{1} x(t): D_{*}^{0.75} x(t)+x(t)=y(t)-\sin t$.

$\Rightarrow p(s)=\mathcal{L}\left[D_{*}^{o 7.5} x(t)+x(t)\right]=s^{0.5}+1$

$\Rightarrow \psi(s)=\frac{1}{p(s)}=\frac{1}{s^{0.75}+1}$

$\Rightarrow u(t)=\mathcal{L}^{-1}[\psi(s)]=t^{-0.25} \sum_{k=0}^{\infty} \frac{\left(-t^{0.75}\right)^{k}}{\Gamma\left(\frac{3 k}{4}+\frac{3}{4}\right)}$

Where the initial approximation must be satisfied by the following equations:

$L_{1} x(t)=0, x(0)=1$.

Therefore, it can be started by:

$$
\begin{aligned}
& x_{0}(t)=v_{1}^{0}=E_{\frac{3}{4}, 1}\left(-t^{o .75}\right)=\sum_{k=0}^{\infty} \frac{\left(-t^{0.75}\right)^{k}}{\Gamma\left(\frac{3 k}{4}+1\right)} \\
& y_{0}(t)=v_{2}^{0}=e^{-t}-\sum_{k=0}^{\infty} \frac{\left(-t^{o .75}\right)^{k}}{\Gamma\left(\frac{3 k}{4}+1\right)}+\sin t
\end{aligned}
$$

Accordingly, by Eq. (5.2) the higher order approximation of the exact solution can be obtained as follows:

$$
\begin{aligned}
& x_{1}(t)=\sum_{i=0}^{1} v_{1}^{k}(t)= \\
& \sum_{k=0}^{\infty} \frac{\left(-t^{o .75}\right)^{k}}{\Gamma\left(\frac{3 k}{4}+1\right)}+ \\
& \int_{0}^{t}\left(\sum_{k=0}^{\infty} \frac{(-1)\left(\tau^{0.75}\right)^{k}}{\Gamma\left(\frac{3 k}{4}+1\right)}\right)\left((t-\tau)^{-0.25} \sum_{k=0}^{\infty} \frac{\left(-(t-\tau)^{0.75}\right)^{k}}{\Gamma\left(\frac{3 k}{4}+\frac{3}{4}\right)}\right)\left(e^{-\tau}-\right. \\
& \left.\sum_{k=0}^{\infty} \frac{\left(-\tau^{0.75}\right)^{k}}{\Gamma\left(\frac{3 k}{4}+1\right)}\right) d \tau
\end{aligned}
$$

\begin{tabular}{|c|c|c|c|c|}
\hline $\mathrm{T}$ & $\begin{array}{c}\mathrm{x}_{1} \\
\alpha=0.5\end{array}$ & $\begin{array}{c}\mathrm{x}_{1} \\
\alpha=0.75\end{array}$ & $\begin{array}{c}\mathrm{x}_{1} \\
\alpha=1\end{array}$ & Exact solution \\
\hline 0 & 1 & 1 & 1 & 1 \\
\hline 0.1 & 0.743 & 0.828 & 0.9048374 & 0.9048374 \\
\hline 0.2 & 0.695 & 0.742 & 0.8187308 & 0.8187308 \\
\hline 0.3 & 0.682 & 0.683 & 0.7408182 & 0.7408182 \\
\hline 0.4 & 0.576 & 0.643 & 0.6703201 & 0.6703201 \\
\hline 0.5 & 0.508 & 0.557 & 0.6065307 & 0.6065307 \\
\hline 0.6 & 0.472 & 0.52 & 0.5488116 & 0.5488116 \\
\hline 0.7 & 0.315 & 0.432 & 0.41965853 & 0.41965853 \\
\hline 0.8 & 0.252 & 0.44 & 0.449329 & 0.449329 \\
\hline 0.9 & 0.187 & 0.327 & 0.4065697 & 0.4065697 \\
\hline 1 & 0.119 & 0.272 & 0.3678794 & 0.3678794 \\
\hline
\end{tabular}

$$
\begin{aligned}
& y_{1}(t)= \\
& \sum_{i=0}^{1} v_{2}^{k}(t)=e^{-t}-\sum_{k=0}^{\infty} \frac{\left(-t^{o .75}\right)^{k}}{\Gamma\left(\frac{3 k}{4}+1\right)}-\int_{0}^{t}\left(\sum_{k=0}^{\infty} \frac{(-1)\left(\tau^{o .75}\right)^{k}}{\Gamma\left(\frac{3 k}{4}+1\right)}\right)((t- \\
& \left.\tau)^{-0.25} \sum_{k=0}^{\infty} \frac{\left(-(t-\tau)^{0.75}\right)^{k}}{\Gamma\left(\frac{3 k}{4}+\frac{3}{4}\right)}\right)\left(e^{-\tau}-\sum_{k=0}^{\infty} \frac{\left(-\tau^{o .75}\right)^{k}}{\Gamma\left(\frac{3 k}{4}+1\right)}\right) d \tau+\sin t
\end{aligned}
$$

Table 1: Numerical Results of the Solution in Example 5.1

Table 1 shows the approximate solutions for Eq. (5.2) obtained for different values of $\alpha$ using our method. The results are in good agreement with the results of the exact solutions.

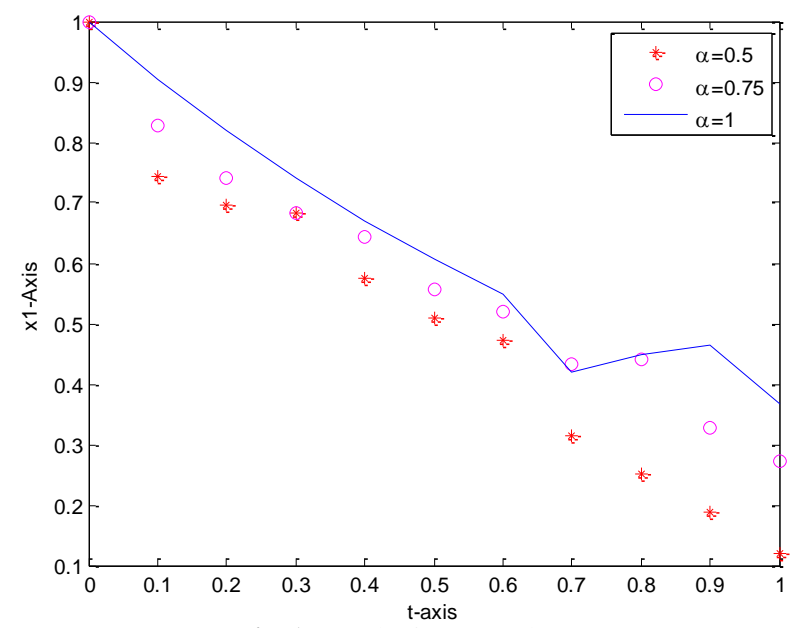

Fig. 1: Results for Example 1.

Example 2: consider the following system of nonlinear fractional order differential -algebraic equations [11]

$$
\left.\begin{array}{c}
D_{*}^{\alpha} x(t)-x(t)+z(t) x(t)=1 \\
D_{*}^{\alpha} z(t)+z(t)-y(t)+x^{2}(t)=0 \\
y(t)-x^{2}(t)=0, t \in[o, 1], 0<\alpha \leq 1
\end{array}\right\}
$$

Subject to initial conditions $\mathrm{x}(0)=\mathrm{y}(0)=\mathrm{z}(0)=10$. For the special case of $\alpha=1$, we have analytical solution $(t)=e^{t}, y(t)=$ $e^{2 t}$, and $z(t)=e^{-t}$.

Solution:

From the Eq. (5.3), optimal selection auxiliary linear operator the equation is represented as follows:

$L_{1} x(t): D_{*}^{\alpha} x(t)-x(t)=1-z(t) x(t)$

$L_{2} z(t): D_{*}^{\alpha} z(t)+z(t)=y(t)-x^{2}(t)$

Therefore $\emptyset\left(v_{i}^{k}\right)$ is defined as:

$$
\left.\begin{array}{c}
\emptyset_{1}\left(v_{1}^{k}, v_{2}^{k}, v_{3}^{k}\right)=\int_{0}^{t} u(t-\tau)\left[1-v_{2}^{k} v_{1}^{k}\right] d \tau \\
\emptyset_{2}\left(v_{1}^{k}, v_{2}^{k}, v_{3}^{k}\right)=\int_{0}^{t} u(t-\tau)\left[v_{3-}^{k} v_{1}^{k}\right] d \tau
\end{array}\right\}
$$

Then, using Eq. (5.4), the Laplace Iteration Method with Lagrange Multiplier formulae in t-direction for the calculation of the approximate solution of Eq. (5.3) can be readily obtained as: 


$$
\left.\begin{array}{c}
x_{n+1}(t)=x_{0}(t) \\
+\int_{0}^{t}\left[\lambda(\tau) u(t-\tau)\left(1-z_{n}(\tau) x_{n}(\tau)\right] d \tau\right. \\
z_{n+1}(t)=z_{0}(t) \\
+\int_{0}^{t}\left[\lambda(\tau) u(t-\tau)\left(y_{n}(\tau)-x_{n}^{2}(\tau)\right] d \tau,\right. \\
y_{n+1}=x_{n+1}^{2}(t)
\end{array}\right\}
$$

Case 1: $\alpha=1$

$$
\begin{aligned}
& L_{1} x(t): D_{*}^{1} x(t)-x(t)=1-z(t) x(t) \\
& \Rightarrow p(s)=\mathcal{L}\left[D_{*}^{1} x(t)-x(t)\right]=s-1 \\
& \Rightarrow \psi(s)=\frac{1}{p(s)}=\frac{1}{s-1} \\
& \Rightarrow u(t)=\mathcal{L}^{-1}[\psi(s)]=e^{t} \\
& u(t-\tau)=e^{(t-\tau)}
\end{aligned}
$$

And

$L_{2} z(t): D_{*}^{1} z(t)+z(t)=y(t)-x^{2}(t)$

$\Rightarrow p(s)=\mathcal{L}\left[D_{*}^{1} z(t)+z(t)\right]=s+1$

$\Rightarrow \psi(s)=\frac{1}{p(s)}=\frac{1}{s+1}$

$\Rightarrow u(t)=\mathcal{L}^{-1}[\psi(s)]=e^{-t}$

$u(t-\tau)=e^{(\tau-t)}$

Where the initial approximation must be satisfied by the following equations:

$L_{1} x(t)=0, x(0)=1 \Longrightarrow x_{0}(t)=v_{1}^{0}=e^{t}$

$L_{2} z(t)=0, z(0)=1 \Longrightarrow z_{0}(t)=v_{2}^{0}=e^{-t}$

$y_{0}(t)=v_{3}^{0}=e^{2 t}$

Accordingly, by Eq. (5.5) the higher order approximation of the exact solution can be obtained as follows:

$x_{1}(t)=\sum_{i=0}^{1} v_{1}^{k}(t)=e^{t}$

$z_{1}(t)=\sum_{i=0}^{1} v_{2}^{k}(t)=e^{-t}$

The remaining approximations $x_{n}=0, z_{n}=0, n>1$ can be completely determined such that each term will be determined using the prevous term: thus, the exact solution is as follows:

$x(t)=\lim _{p \rightarrow \infty} \sum_{i=0}^{p} v_{1}^{k}(t)=e^{-t}$

$z(t)=\lim _{p \rightarrow \infty} \sum_{i=0}^{p} v_{2}^{k}(t)=e^{t}$

$y(t)=\lim _{p \rightarrow \infty} \sum_{i=0}^{p} v_{3}^{k}(t)=e^{2 t}$

\section{Case 1: $\alpha=0.5$}

$L_{1} x(t): D_{*}^{0.5} x(t)-x(t)=1-z(t) x(t)$

$\Longrightarrow p(s)=\mathcal{L}\left[D_{*}^{o .5} x(t)-x(t)\right]=s^{0.5}-1$

$\Longrightarrow \psi(s)=\frac{1}{p(s)}=\frac{1}{s^{0.5}-1}$

$\Rightarrow u(t)=\mathcal{L}^{-1}[\psi(s)]=t^{-0.5} \sum_{k=0}^{\infty} \frac{(\sqrt{t})^{k}}{\Gamma\left(\frac{k}{2}+\frac{1}{2}\right)}$
And

$L_{2} z(t): D_{*}^{0.5} z(t)+z(t)=y(t)-x^{2}(t)$

$\Rightarrow p(s)=\mathcal{L}\left[D_{*}^{0.5} z(t)+z(t)\right]=s^{0.5}+1$

$\Rightarrow \psi(s)=\frac{1}{p(s)}=\frac{1}{s^{0.5}+1}$

$\Rightarrow u(t)=\mathcal{L}^{-1}[\psi(s)]=t^{-0.5} \sum_{k=0}^{\infty} \frac{(-\sqrt{t})^{k}}{\Gamma\left(\frac{k}{2}+\frac{1}{2}\right)}$

Where the initial approximation must be satisfied by the following equations:

$L_{1} x(t)=0, x(0)=1 \Rightarrow x_{0}(t)=v_{1}^{0}=E_{\frac{1}{2}, 1}(\sqrt{t})=\sum_{k=0}^{\infty} \frac{(\sqrt{t})^{k}}{\Gamma\left(\frac{k}{2}+1\right)}$

$L_{2} z(t)=0, z(0)=1 \Rightarrow z_{0}(t)=v_{2}^{0}=E_{\frac{1}{2}, 1}(-\sqrt{t})=\sum_{k=0}^{\infty} \frac{(-\sqrt{t})^{k}}{\Gamma\left(\frac{k}{2}+1\right)}$

$y_{0}(t)=v_{3}^{0}=\left(\sum_{k=0}^{\infty} \frac{(\sqrt{t})^{k}}{\Gamma\left(\frac{k}{2}+1\right)}\right)^{2}$

Accordingly, by Eq. (5.5) the higher order approximation of the exact solution can be obtained as follows:

$x_{1}(t)=\sum_{i=0}^{1} v_{1}^{k}(t)=\sum_{k=0}^{\infty} \frac{(\sqrt{t})^{k}}{\Gamma\left(\frac{k}{2}+1\right)}+\int_{0}^{t}\left(\sum_{k=0}^{\infty} \frac{\left((-1)^{k+1} \tau^{\frac{k}{2}}\right.}{\Gamma\left(\frac{k}{2}+1\right)}\right)((t-$

$\left.\tau)^{-0.5} \sum_{k=0}^{\infty} \frac{(-1)^{k}(t-\tau)^{\frac{k}{2}}}{\Gamma\left(\frac{k}{2}+\frac{1}{2}\right)}\right)\left(1-\left(\sum_{k=0}^{\infty} \frac{(\sqrt{\tau})^{k}}{\Gamma\left(\frac{k}{2}+1\right)}\right) \sum_{k=0}^{\infty} \frac{(-\sqrt{\tau})^{k}}{\Gamma\left(\frac{k}{2}+1\right)}\right) d \tau$

$z_{1}(t)=\sum_{i=0}^{1} v_{2}^{k}(t)=\sum_{k=0}^{\infty} \frac{(-\sqrt{t})^{k}}{\Gamma\left(\frac{k}{2}+1\right)}+\int_{0}^{t}\left(\sum_{k=0}^{\infty} \frac{(-1) \tau^{\frac{k}{2}}}{\Gamma\left(\frac{k}{2}+1\right)}\right)((t-$

$\left.\tau)^{-0.5} \sum_{k=0}^{\infty} \frac{(-1)^{k}(\tau-t)^{\frac{k}{2}}}{\Gamma\left(\frac{k}{2}+\frac{1}{2}\right)}\right)\left(\left(\sum_{k=0}^{\infty} \frac{(\sqrt{\tau})^{k}}{\Gamma\left(\frac{k}{2}+1\right)}\right)^{2}-\left(\sum_{k=0}^{\infty} \frac{(\sqrt{\tau})^{k}}{\Gamma\left(\frac{k}{2}+1\right)}\right)^{2}\right) d \tau$

$y_{1}(t)=\sum_{i=0}^{1} v_{3}^{k}(t)=\left(x_{1}(t)\right)^{2}$

Case 1: $\alpha=0.75$

$L_{1} x(t): D_{*}^{0.75} x(t)-x(t)=1-z(t) x(t)$

$\Rightarrow p(s)=\mathcal{L}\left[D_{*}^{o .75} x(t)-x(t)\right]=s^{0.5}-1$

$\Rightarrow \psi(s)=\frac{1}{p(s)}=\frac{1}{s^{0.75}-1}$

$\Rightarrow u(t)=\mathcal{L}^{-1}[\psi(s)]=t^{-0.25} \sum_{k=0}^{\infty} \frac{\left(t^{0.75}\right)^{k}}{\Gamma\left(\frac{3 k}{4}+\frac{3}{4}\right)}$

And

$L_{2} z(t): D_{*}^{0.75} z(t)+z(t)=y(t)-x^{2}(t)$

$\Rightarrow p(s)=\mathcal{L}\left[D_{*}^{07.5} z(t)+z(t)\right]=s^{0.5}+1$

$\Rightarrow \psi(s)=\frac{1}{p(s)}=\frac{1}{s^{0.75}+1}$

$\Rightarrow u(t)=\mathcal{L}^{-1}[\psi(s)]=t^{-0.25} \sum_{k=0}^{\infty} \frac{\left(-t^{0.75}\right)^{k}}{\Gamma\left(\frac{3 k}{4}+\frac{3}{4}\right)}$

Where the initial approximation must be satisfied by the following equations:

$L_{1} x(t)=0, x(0)=1 \Rightarrow x_{0}(t)=v_{1}^{0}=E_{\frac{3}{4}, 1}\left(t^{o .75}\right)=\sum_{k=0}^{\infty} \frac{\left(t^{0.75}\right)^{k}}{\Gamma\left(\frac{3 k}{4}+1\right)}$

$L_{2} z(t)=0, z(0)=1 \Rightarrow z_{0}(t)=v_{2}^{0}=E_{\frac{3}{4}, 1}\left(-t^{0.75}\right)$ 
$=\sum_{k=0}^{\infty} \frac{\left(-t^{o .75}\right)^{k}}{\Gamma\left(\frac{3 k}{4}+1\right)}$

$y_{0}(t)=v_{3}^{0}=\left(\sum_{k=0}^{\infty} \frac{\left(t^{0.75}\right)^{k}}{\Gamma\left(\frac{3 k}{4}+1\right)}\right)^{2}$

Accordingly, by Eq. (5.5) the higher order approximation of the exact solution can be obtained as follows:

$x_{1}(t)=\sum_{i=0}^{1} v_{1}^{k}(t)=$

$\sum_{k=0}^{\infty} \frac{\left(t^{o .75}\right)^{k}}{\Gamma\left(\frac{3 k}{4}+1\right)}+$

$\int_{0}^{t}\left(\sum_{k=0}^{\infty} \frac{(-1)^{k+1}\left(\tau^{0.75}\right)^{k}}{\Gamma\left(\frac{3 k}{4}+1\right)}\right)\left((\tau-t)^{-0.25} \sum_{k=0}^{\infty} \frac{\left((\mathrm{t}-\tau)^{0.75}\right)^{\mathrm{k}}}{\Gamma\left(\frac{3 \mathrm{k}}{4}+\frac{3}{4}\right)}\right)(1-$

$\left.\left(\sum_{\mathrm{k}=0}^{\infty} \frac{\left(\tau^{0.75}\right)^{\mathrm{k}}}{\Gamma\left(\frac{3 \mathrm{k}}{4}+1\right)}\right) \sum_{\mathrm{k}=0}^{\infty} \frac{\left(-\tau^{0.75}\right)^{\mathrm{k}}}{\Gamma\left(\frac{3 \mathrm{k}}{4}+1\right)}\right) \mathrm{d} \tau$

$\mathrm{z}_{1}(\mathrm{t})=\sum_{\mathrm{i}=0}^{1} \mathrm{v}_{2}^{\mathrm{k}}(\mathrm{t})=\sum_{\mathrm{k}=0}^{\infty} \frac{\left(\tau^{0.75}\right)^{\mathrm{k}}}{\Gamma\left(\frac{\mathrm{k} k}{4}+1\right)}+\int_{0}^{\mathrm{t}}\left(\sum_{\mathrm{k}=0}^{\infty} \frac{(-1)\left(\mathrm{\tau}^{0.75}\right)^{\mathrm{k}}}{\Gamma\left(\frac{\mathrm{k} k}{4}+1\right)}\right)((\mathrm{t}-$

$\left.\tau)^{-0.25} \sum_{\mathrm{k}=0}^{\infty} \frac{\left(-\tau^{0.75}\right)^{\mathrm{k}}}{\Gamma\left(\frac{3 \mathrm{k}}{4}+\frac{3}{4}\right)}\right)\left(\left(\sum_{\mathrm{k}=0}^{\infty} \frac{\left(\tau^{0.75}\right)^{\mathrm{k}}}{\Gamma\left(\frac{3 \mathrm{k}}{4}+1\right)}\right)^{2}-\left(\sum_{\mathrm{k}=0}^{\infty} \frac{\left(\tau^{0.75}\right)^{\mathrm{k}}}{\Gamma\left(\frac{3 \mathrm{k}}{4}+1\right)}\right)^{2}\right) \mathrm{d} \tau$

$\mathrm{y}_{1}(\mathrm{t})=\sum_{\mathrm{i}=0}^{1} \mathrm{v}_{3}^{\mathrm{k}}(\mathrm{t})=\left(\mathrm{x}_{1}(\mathrm{t})\right)^{2}$

Table 2: Numerical Results of the Solution in Example 5.2

\begin{tabular}{lllll}
\hline $\mathrm{T}$ & \multicolumn{1}{c}{$\mathrm{x}_{1}$} & \multicolumn{1}{c}{$\mathrm{x}_{1}$} & \multicolumn{1}{c}{$\mathrm{x}_{1}$} & Exact solution \\
\hline 0 & 1 & 1 & $\alpha=0.75$ & 1 \\
0.1 & 1.357 & 1.193 & 1.1051709 & 1.1051709 \\
0.2 & 1.505 & 1.325 & 1.2214027 & 1.2214027 \\
0.3 & 1.618 & 1.441 & 1.3498588 & 1.3498588 \\
0.4 & 1.714 & 1.547 & 1.4918246 & 1.4918246 \\
0.5 & 2.564 & 1.913 & 1.6487212 & 1.6487212 \\
0.6 & 2.824 & 2.091 & 1.8221188 & 1.8221188 \\
0.7 & 3.085 & 2.273 & 2.0137527 & 2.0137527 \\
0.8 & 3.348 & 2.495 & 2.2255409 & 2.2255409 \\
0.9 & 2.97 & 2.648 & 2.4596031 & 2.4596031 \\
1 & 3.128 & 2.84 & 2.7182818 & 2.7182818 \\
\hline
\end{tabular}

Table 2 shows the approximate solutions for Eq. (5.5) obtained for different values of $\alpha$ using our method. The results are in good agreement with the results of the exact solutions.

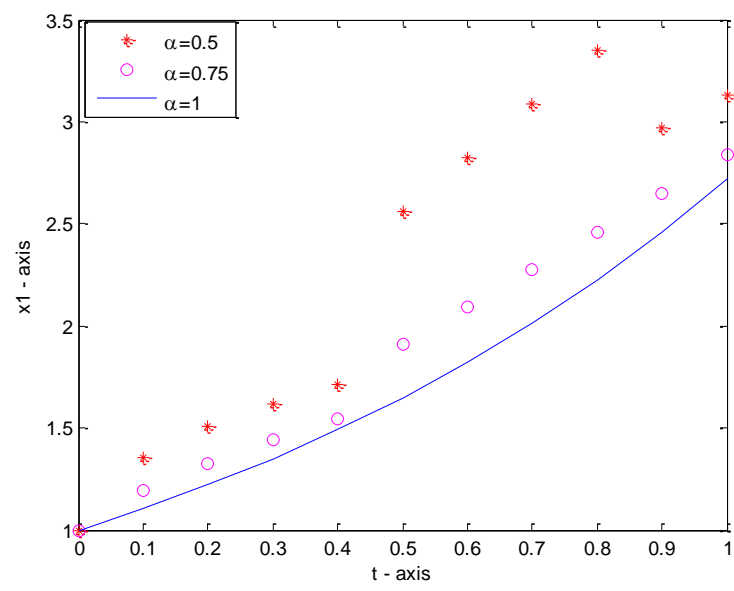

Fig. 2: Results for Example 2.

\section{Discussion}

In this study, the present method (LLIM) has been extended to solve fractional order differential-algebraic equations (FDAEs) Two examples are given to demonstrate to powerfulness of the method. The results obtained by the method are in good agreement with the exact solution. The study shows that the method is a reliable technique to solve fractional differential-algebraic equations, and often notable advantages from the points of applicability, computational costs, and accuracy.

\section{References}

[1] J.D. Munkhammar, Fractional Caluslus and the Taylor-Riemann Series, Undergraduate Math. Journal, 6(2005) 1-19.

[2] I. Podlubny, Fractional differential equations: an introduction to fractional derivative, fractional differential equations, to methods of their solution and some of their applications, a cademic press, Now York, 1999.

[3] M. Caputo, Linear models of dissipation whose Q is almost frequency independent, part П. J Roy Austral Soc., 13(1967) 529-539. http://dx.doi.org/10.1111/j.1365-246X.1967.tb02303.x.

[4] S.G. Samko., A.A. Kilbas A. A., O.I./marichev, Fractional Integrals and Derivatives: Theory and Applications, Gordon and Breach, London, 1993.

[5] F. Mainardi, Fractional Calculus: Some basic Problems in continuum and Statistical Mechanics, in Fractals and Fractional Calculus in Continuum Mechanics, Vol. 378 of CISM Courses and Lectures, pp. 291-348, Springer Vienna Austria, 1997.

[6] R. Gorenflo., F. Mainardi, Fractional Calculus: Integral and Differential Equations of Fractional Order", Fractals and Fractional Calculus in Continuum Mechanics, Vol. 378, of CISM Courses and Lectures, pp. 223-276, Springer, Vienna, Austria, 1997.

[7] B.Ibis B., M. Bayram, Numerical Comparsion of Methods for Solving Fractional differential-Algebraic Equations (FDAEs), Comput. $\begin{array}{lll}\text { Math. } & \text { Appl., } & \text { 62(2011) }\end{array}$ http://dx.doi.org/10.1016/j.camwa.2011.08.043.

[8] B.Ibis, M. Bayram, A.G. Agargun, Applicationa of Fractional Transform Method to Fractional Differential-Algebraic Equations, Eur. J. Pure. Appl. Math, 4 (2011) 129-141.

[9] M.Zurigat, S. Momani , Alawneh, Analytical Approximate Solutions of Systems of Fractional algebraic Differential Equations by Homotopy Analysis Method, Comput. Math. Appl., 59(2010) 12271235. http://dx.doi.org/10.1016/j.camwa.2009.07.002.

[10] X.L.Ding, Y.L. Jiang, Waveform Relaxation Methods for Frational Differential-Algebraic Equations, Fract. Calc. Appl. Anal, 17(2014) 585-604. http://dx.doi.org/10.2478/s13540-014-0187-z.

[11] S.K.Damarla , K. Kundu , Numerical Solution of Fractional Order Differential-Agebraic Equations Using Generalized Triangular Function Operational Matrices, Journal of Fractional Calculus and Applications, 6(July 2015)31-52, No. 2.

[12] B.Barani,S.M. Hosseini., M.Saffarzadeh, S. Javanmardi S., Analytical Approach of Differential-Algebraic Equations of Fractional Order Via Homotopy Perturbation, Communications on Adanced Computational Science with Applications, 2014(2014)1-8. http://dx.doi.org/10.5899/2014/cacsa-00013.

[13] Hebibolla Latifizadeh, Esmail Hesameddini, Flexibility and Efficiency of New Analytical Method for Solving Systems of Linear and Nonlinear Differential Equations, International Journal of Science and Engineering Investigations, 2(July 2013) 44-51.

[14] I. Podlubny, Fractional Differential Equations, A cademic Press, 1999.

[15] A.A.Kilbas, M. Saigo, R.K.Saxena, Generalized Mittag-Leffler Function and Generalized Fractional Calculus Operators, Integral Transforms Special Func, 1592004) 31-49.

[16] JH He, Variational Iteration Method for Autonomous Ordinary Differential, App. Math. Comput, 114(2000)115-123. http://dx.doi.org/10.1016/S0096-3003(99)00104-6.

[17] JH He, Variational Principle for Some Nonlinear Partial Differential Equations with Variable Coefficients, Chaos Solitions Fractals, 19(2004)847-851. http://dx.doi.org/10.1016/S0960-0779(03)002650.

[18] JH He, Variational Iteration Method Some Recents and New Interpretations, J. Comp. Appl. Math., 367 (2007) 188-191. http://dx.doi.org/10.1016/i.cam.2006.07.009.

[19] JH. He, SQ.Wang, Variational Iteration Method for Solving Integro-Differential Equations, Phys. Lett. A., 207(2007) 3-17. 